\title{
Multidisciplinary Management of Multiple Sclerosis Symptoms
}

\author{
Philippe Gallien ${ }^{\mathrm{a}} \quad$ Jordi Gich $^{\mathrm{b}}$ Bernardo F. Sánchez-Dalmau ${ }^{\mathrm{c}}$ \\ Wolfgang Feneberg ${ }^{\mathrm{d}}$ \\ aPôle MPR Saint Helier Rennes MS Clinic, Rennes, France; ${ }^{b}$ Dr. Josep Trueta University Hospital of Girona, Girona;

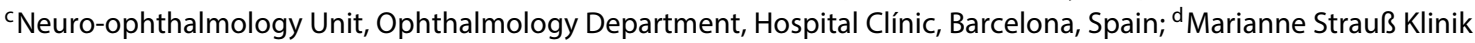 \\ Berg, Bavaria, Germany
}

\section{Key Words}

Multidisciplinary care $\cdot$ Multiple sclerosis .

Neurology-pain · Neuro-ophthalmology ·

Neuropsychology · Neurorehabilitation

\begin{abstract}
Background: Effective management of multisymptomatic chronic diseases such as multiple sclerosis (MS) requires a multimodal, interdisciplinary approach. At MS clinics, numerous healthcare specialties are coordinated to provide patients with quality clinical care for all aspects of their disease. Settings and resource availability may vary between countries. Four specific specialty services from different EU countries are examined in more detail. Summary: The multidisciplinary neurorehabilitation team in Rennes, France, provides specialized consultations (e.g. spasticity, urodynamic unit, devices), inpatient and outpatient intensive rehabilitation programs and therapeutic education. Management approaches are based on a patient's level of impairment as assessed by the Expanded Disability Status Scale. In Girona, Spain, neuropsychologists perform assessments as part of the neurological protocol for all patients with MS. Depending on the level of impairment and patients' characteristics (e.g. working or not working), cognitive deficits may be treated at home or at a neurorehabilitation center. In Barcelona, Spain, neuro-ophthalmologists are involved in the differential diagnosis and follow-up care of MS patients with visual distur-
\end{abstract}

bances; particular attention is given to patients' vision-related quality of life. Pain specialists at the Marianne Strauß Klinik in Berg, Germany, have developed a system for classifying MS pain syndromes and differentiating MS-related pain from non MS-related pain. Chronic pain management involves numerous disciplines and requires active engagement by patients in developing treatment plans. Key Messages: MS affects several body systems and patients invariably require specialized interdisciplinary support. Insight into services provided by various specialties and their fit within multidisciplinary care models at MS centers may facilitate the design or refinement of care models in other locations.

(c) 2014 S. Karger AG, Base

\section{Physical Medicine and Rehabilitation in France \\ Philippe Gallien \\ E-Mail philippe.gallien@pole-sthelier.com}

Management of neurological deficits in patients with multiple sclerosis (MS) involves collaboration between neurologists, occupational therapists, speech therapists, physiotherapists and other allied specialties. Physical medicine and rehabilitation play an important role.

At the Saint Helier Center for Physical Medicine and Rehabilitation in Rennes, France, the MS clinic is an important area of activity for neurorehabilitation. In conjunction with the neurologist, team members provide counselling and develop proposals for the management of various impairments. The main area of activity of neurorehabilitation, however, is in the rehabilitation center where

\section{KARGER}

E-Mail karger@karger.com www.karger.com/ene (c) 2014 S. Karger AG, Basel

0014-3022/14/0721-0020\$39.50/0
Philippe Gallien

Pôle MPR Saint Helier Rennes MS Clinic

54, rue Saint-Hélier, FR-35043 Rennes Cedex (France)

E-Mail philippe.gallien@pole-sthelier.com 
the team provides specialized consultations (e.g. spasticity, urodynamic unit, devices), inpatient and outpatient intensive rehabilitation programs and therapeutic education.

In 2013, 350 patients visited the Saint Helier Center; 260 patients benefited from rehabilitation programs and 70 patients benefited from a specific spasticity consultation (e.g. botulinum toxin in focal cases, intrathecal baclofen in refractory cases).

MS patients are assessed regularly to evaluate their disease evolution. The management approach is based on an individual's level of impairment as measured on the Expanded Disability Status Scale (EDSS) $[1,2]$.

\section{Managing Patients with a Lower EDSS Score (0-3)}

Patients with an EDSS score of $0-3$ are usually managed as outpatients. In the absence of any physical impairment, information and therapeutic education is provided on request. Mild impairment tends to involve specific management of one or two symptoms such as spasticity, urinary disorders or mild gait/posture disturbances. External therapists provide counselling and encouragement for patients to maintain regular physical activities.

\section{Managing Patients with a Medium EDSS Score (3-6)}

A greater degree of disability (EDSS score 3-6) is frequently associated with social and professional consequences on a patient's daily life. Management involves either inpatient or outpatient programs led by a multidisciplinary team consisting of a physiotherapist, speech therapist, occupational therapist and/or neuropsychologist. The aim of management is to achieve functional recovery (e.g. physical training, muscle strengthening) so that patients can continue with their activities. Fatigue is a common complaint at this level of disability. Adaptations to the patient's environment and daily activities may be required to lessen the impact of fatigue. Technical aids such as a wheelchair, crutches and ankle foot orthoses may also be discussed. The neurorehabilitation team liaises regularly with the patient's external therapist to encourage and facilitate maintenance of physical and social activities.

\section{Managing Patients with a high EDSS Score (>6)}

In patients with an EDSS score $>6$, the main goals of physical therapy and rehabilitation are to maintain patients' autonomy and quality of life at home and to avoid
Table 1. Neuropsychological aspects of multiple sclerosis

\begin{tabular}{ll}
\hline Cognition & $\begin{array}{l}\text { Processing speed; working memory and } \\
\text { other executive functions; memory } \\
\text { disorders (mainly information recovery) }\end{array}$ \\
\hline Mood disorders & $\begin{array}{l}\text { Affective disorders and adaptive } \\
\text { disorders }\end{array}$ \\
\hline Behavioral symptoms & Apathy, fatigue and loss of inhibition \\
\hline
\end{tabular}

complications such as pressure sores, respiratory failure and/or urinary complications. Inpatient management tends to focus largely on occupational therapy and disability compensation, but also with a view towards functional recovery. At this level of impairment, invasive therapies such as an intrathecal baclofen pump for refractory spasticity, Bricker surgery for urinary disorders or gastrostomy for swallowing disorders often need to be considered. Patients receive home care from a nurse and physiotherapist. The majority of patients with high EDSS scores require technical aids and are reliant on considerable support from their carers.

\section{Neuropsychology \\ Jordi Gich \\ E-Mail jgich.girona.ics@gencat.cat}

The MS unit at the Dr Josep Trueta University in Girona, Spain, provides care for 700 patients and performs approximately 150 neuropsychological assessments each year. The MS unit team consists of neurologists, neuropsychologists, registered nurses, biochemists and a secretary. The MS unit is located in a general hospital with access to the complete range of medical specialties. At another site, the team works in tandem with a full rehabilitation service.

A neuropsychological assessment is included in the neurological protocol for all patients with MS (fig. 1). The neuropsychological aspects of MS can be classified into three broad categories: cognition, mood disorders and behavioral symptoms (table 1). At a patient's first visit to the unit, an assessment is performed prior to the commencement of immune therapy. This baseline examination is essential to evaluate the evolution of cognitive impairment. Several versions of Rao's battery of neuropsychological tests are used for assessment. The examination is repeated after 3 years or as needed if any substantial change in cognition is suspected.

If a neuropsychological diagnosis is made in the early stages of MS, the symptom category determines the type of treatment administered. In the case of mood disorders (e.g. depression) or behavioral changes, patients with a mild deficit can often be managed internally in conjunction with the neurologist whereas those with a severe deficit are referred to a psychiatrist.

Cognition is the domain of the neuropsychologist. If cognitive impairment is detected, the action taken depends on the patient's 


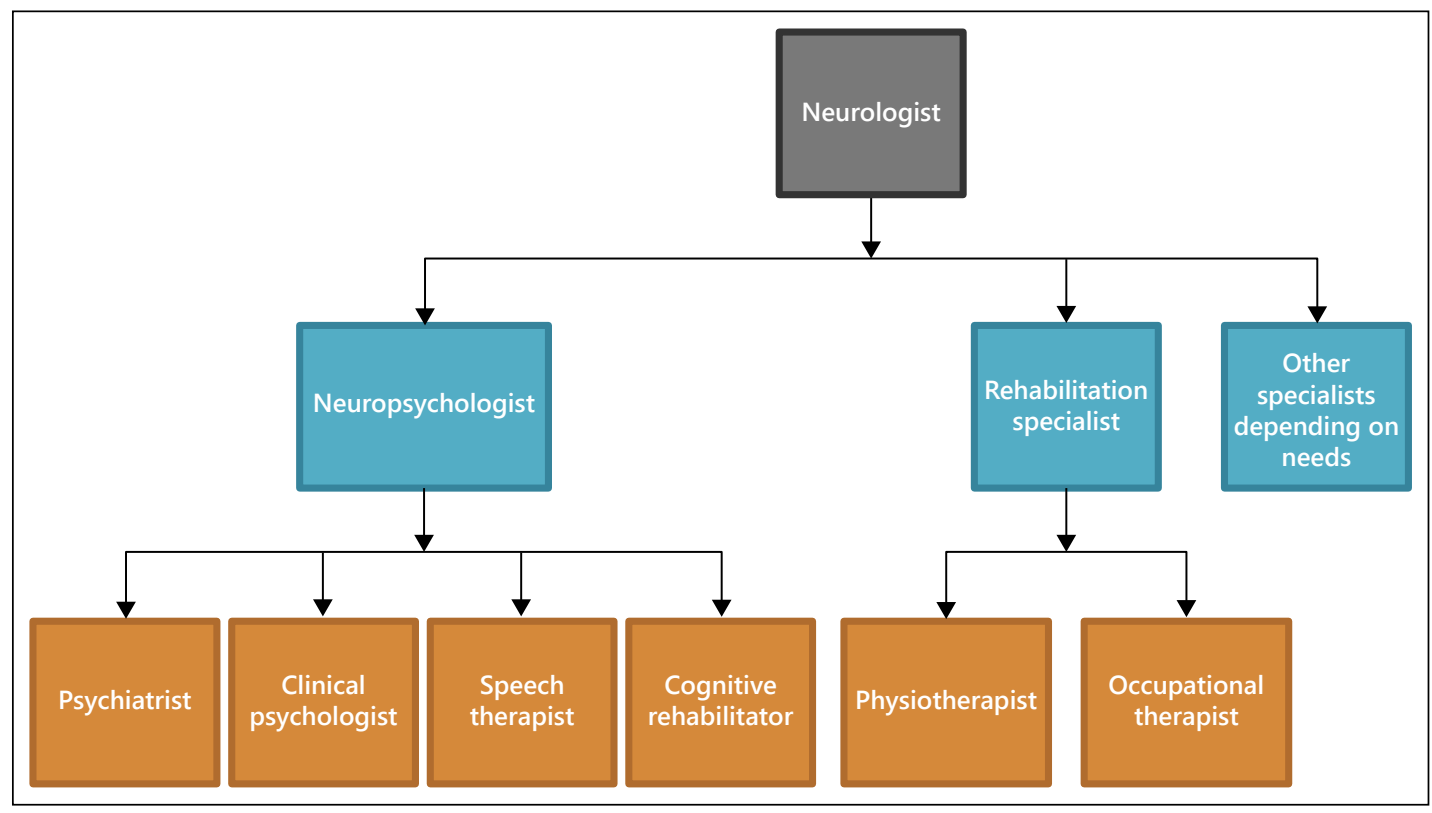

Fig. 1. Multidisciplinary management of patients with multiple sclerosis at the Dr. Josep Trueta University in Girona, Spain. The neuropsychologist refers patients to other services based on individual needs.

characteristics. Mild deficits tend to involve processing speed and working memory. If the deficit is mild and the patient continues to work, our preference is that patients perform home-based exercises with the participation of their family; the MS unit provides attractive free material for home-based exercises. If the cognitive impairment is mild and the patient does not work, the patient can choose between home-based exercises or referral to a neurorehabilitation center. If the cognitive impairment is moderate or severe, which typically occurs in the later stages of MS, the patient is referred to a neurorehabilitation center.

Irrespective of the severity of the deficit, it is important in all cases of cognitive impairment that regular neuropsychological assessment be conducted (e.g. at the end of immunotherapy, in the event of worsening) to monitor its evolution.

\section{Neuro-Ophthalmology in MS}

\section{Bernardo Sánchez-Dalmau}

\section{E-Mail bsanchezdalmau@gmail.com}

It is estimated that up to $80 \%$ of patients with relapsing-remitting MS have visual disturbances. Neuro-ophthalmological disorders in MS can arise in both the afferent and efferent visual pathways (table 2). In Barcelona, Spain, patients either newly diagnosed or with an established diagnosis of MS who present with an episode of visual loss or double vision are referred to the neuro-ophthalmology unit at the Hospital Clinic for a diagnostic work-up.

In patients with acute or subacute visual loss, a differential diagnosis is performed to distinguish between optic neuritis, other inflammatory optic nerve pathologies and retinal disorders (table 3 ).
Table 2. Neuro-ophthalmological disorders in multiple sclerosis
Afferent visual pathway

Optic neuritis

Pars planitis

Uveitis

Periphlebitis

Retrogeniculate

\section{Efferent visual pathway} Saccadic dysmetria

Internuclear ophthalmoplegia

Nystagmus

Oculomotor palsy
Table 3. Differential diagnosis of acute/subacute visual loss in patients with multiple sclerosis

Optic neuritis

Other optic nerve disorders

Neuromyelitis optica (NMO)

Chronic relapsing inflammatory optic neuropathy (CRION)

Autoimmune disorders

Anterior ischemic optic neuropathy (AION)

Compressive disorders

Retinal disorders

Central serous corioretinopathy

Retinal detachment 
The diagnosis is followed by a treatment indication in consultation with the neurologist and further clinical evaluation. The patient's treatment is coordinated with the MS unit. Follow-up is performed at the appearance of new symptoms or at least once yearly in the early stages of MS.

Some useful ancillary investigative tools for diagnosis and management of MS patients with visual disturbances are the low-contrast visual acuity test, color vision test, optical coherence tomography, computerized perimetry and wide-field retinography. This latter technique permits wide-angle visualization of the entire retina, including peripheral areas, in a non-dilated pupil.

An important aspect in the management of MS patients with visual disturbances is their vision-related quality of life. Our preference is to use vision-specific questionnaires such as the National Eye Institute 25-Item Visual Function Questionnaire (NEI-VFQ-25), the 32-item Impact of Visual Impairment (IVI) questionnaire and the Visual Symptoms and Quality of Life (VSQ) questionnaire as these instruments have been shown to correlate with both structural measurements and functional disability.

\section{Management of Pain in MS \\ Wolfgang Feneberg \\ E-Mail wolfgang.feneberg@ms-klinik.de}

The Marianne Strauß Klinik in Berg, Germany, combines acute care services with intensive rehabilitative services at a single center. In operation since 1988, the clinic is the specialist treatment center for MS patients from across the country with all disability levels (EDSS 0-9.5). About 2,500 patients are attended each year. Facilities include 100 inpatient beds, a daycare clinic (12 places), an outpatient clinic and a nursing home (23 care places).

The clinic's in-house resources cover a wide range of specialty services (note: services highlighted in bold are involved in the management of pain): neurology, neuro-urology, psychiatry, clinical psychology and neuropsychology, neuro-palliative care, internal medicine, radiology (basic), physical therapy and rehabilitation, physiotherapy, occupational therapy, speech therapy and swallowing therapy, and social services. Additional consultative support is provided from neuroradiology, endoscopy, ophthalmology, orthopedics, neurosurgery, dermatology, urology, gynecology and aids adaption and supply.

\section{Features of MS Pain}

Several years ago, we conducted a survey to examine the 1-year prevalence of pain syndromes and quality of care in MS patients [3]. A total of 157 consecutive inpatients, aged 19-85 years, with EDSS scores of $1.0-8.5$ participated in the survey. Using a standardized questionnaire, we documented and classified only those pain episodes of an intensity $\geq 4$ on a $0-10$ visual analogue scale (VAS) which had occurred more often than three times or lasted longer than 1 week within the last year. In all, $61 \%$ of patients reported 176 pain syndromes. The most common pain types were headache $(36 \%)$ and neuropathic pain $(29 \%)$. Some particularly relevant findings of the study were as follows:

- $13 \%$ of patients experienced pain at the beginning of MS.

- $20 \%$ of patients experienced pain during relapses.

- $12 \%$ of patients considered pain the worst symptom of MS.

- $68 \%$ of patients reported insufficient pain care by their consulting physician.

- $40 \%$ of patients reported one pain syndrome and $41 \%$ reported two pain syndromes.

- $35 \%$ of patients reported VAS pain intensity scores of $9-10$.

On the basis of these survey results, we implemented a practical system for classifying pain syndromes (fig. 2) [4].

Although pain is relatively common in MS patients, it is frequently not mentioned during a consultation and physicians need to adopt a pro-active approach. $\mathrm{Pa}$ tients should be asked specifically about each pain type, including headaches, as many believe that headaches are not related to MS. If a new pain syndrome is identified, the next step is to ascertain whether it is related to MS. Sources of pain not directly related to MS can include among others spinal fractures, fractures due to osteoporosis, hip necrosis during corticosteroid therapy, restless legs syndrome in patients with severe spasticity and deep vein thrombosis. When evaluating the efficacy of a pain treatment it is essential to be specific about which pain type is 'better' as patients often confuse pain syndromes. Pain type depends to a large extent on the level of disability. Whereas headache is more common in patients with lower EDSS scores, musculoskeletal/ spasticity pain is associated with medium EDSS scores and neuropathic pain is associated with higher EDSS scores.

At the Marianne Strauß Klinik, chronic pain in MS patients is managed using a combination of medical and nonmedical options (multimodal and interdisciplinary therapy) (fig. 3). The therapeutic cycle begins with the usual medical examination at which time the pain is analyzed, measured, diagnosed and classified. Initial treatment of pain is non-medical, involving mainly nursing and physical therapy; pharmacotherapy tailored to the specific pain syndrome and/or aids may be added as needed. For pain syndromes lasting longer 


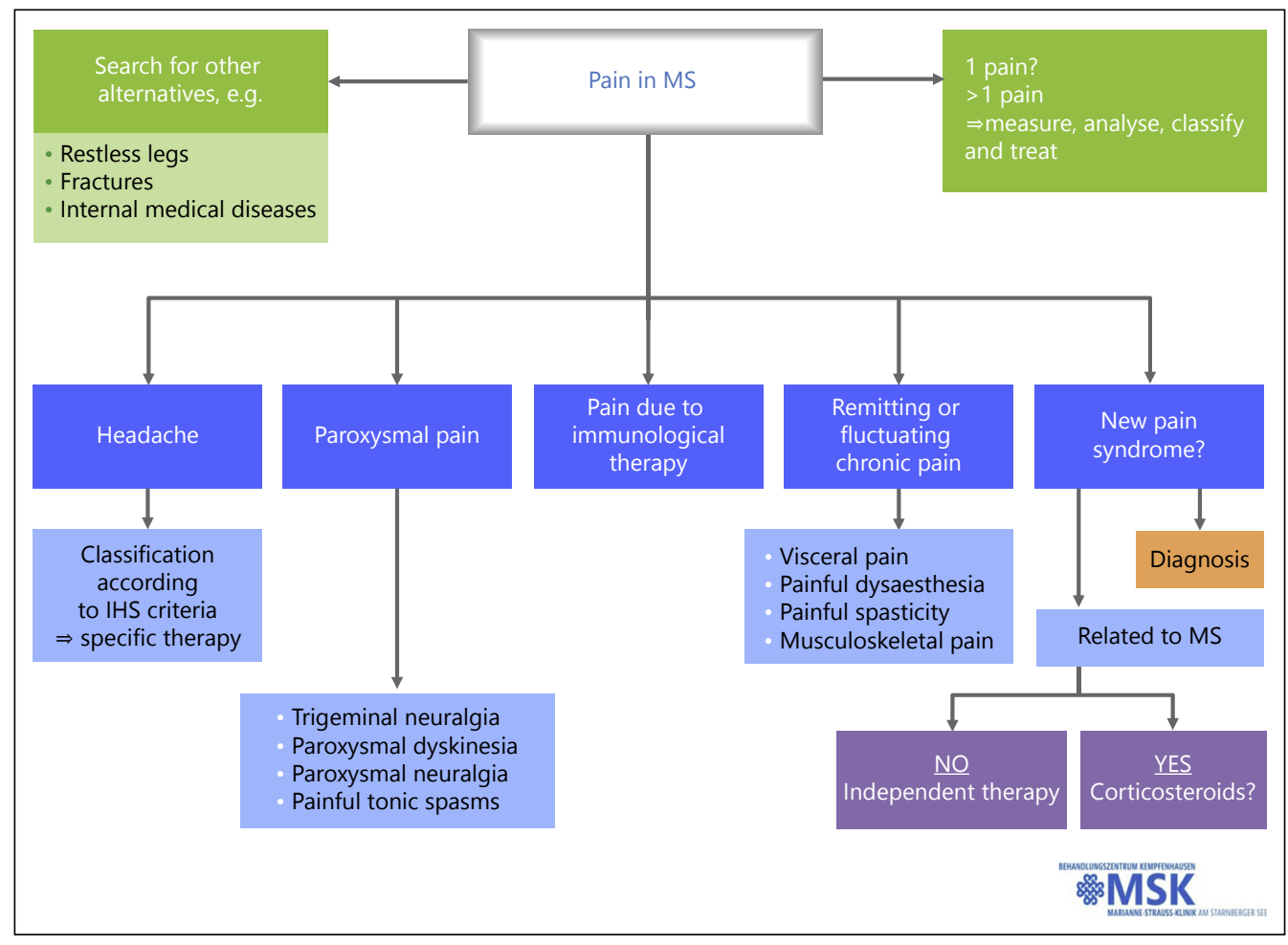

Fig. 2. Classification system for pain syndromes in patients with multiple sclerosis (MS) at the Marianne Strauß Klinik in Berg, Germany.

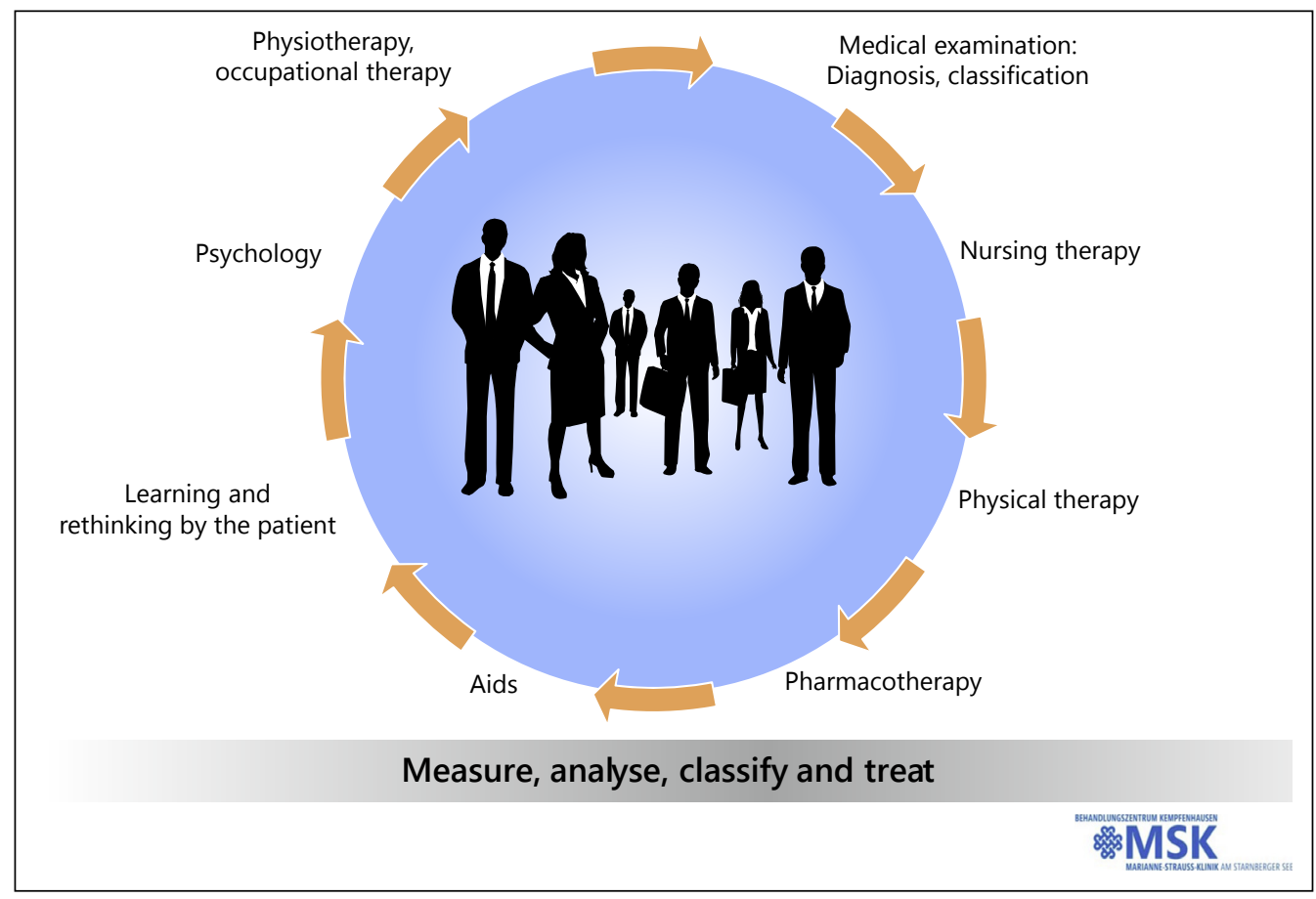

Fig. 3. Multimodal and interdisciplinary approach to treatment of chronic pain in patients with multiple sclerosis at the Marianne Strauß Klinik in Berg, Germany. 
than 3 months, pharmacotherapy alone is ineffective. Effective management of chronic pain requires active learning and rethinking by the patient. To this end, psychological support, as well as physiotherapy and occupational therapy, may be useful for some patients.

\section{Disclosures/Conflict of Interest}

PG has received honoraria from Allergan, Almirall SA and Novartis. JG, BFS and WF have received honoraria from Almirall SA.

Writing assistance was provided by Content Ed Net (Madrid, Spain), with funding from Laboratorios Almirall SA (Barcelona, Spain).

\section{References}

1 Gallien P, Nicolas B, Robineau S, Pétrilli S, Houedakor J, Durufle A: Physical training and multiple sclerosis. [Article in English, French]. Ann Readapt Med Phys 2007;50: 373-376, 369-372.
2 Demaille-Wlodyka S, Donze C, Givron P, Gallien P; ETP Sofmer Group: Self care programs and multiple sclerosis: physical therapeutics treatment - literature review [Article in English, French]. Ann Phys Rehabil Med 2011;54:109-128.

3 Pöllmann W, Feneberg W, Erasmus LP: Pain in multiple sclerosis-a still underestimated problem. The 1 year prevalence of pain syndromes, significance and quality of care of multiple sclerosis inpatients [Article in German]. Nervenarzt 2004;75:135-140.
-4 Pöllmann W, Feneberg W: Current management of pain associated with multiple sclerosis. CNS Drugs 2008;22:291-324. 